Research Paper

\title{
Drug-drug interaction of acetaminophen and roxithromycin with the cocktail of cytochrome P450 and hepatotoxicity in rats
}

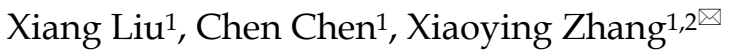 \\ 1. Chinese-German Joint Laboratory for Natural Product Research, College of Biological Science and Engineering, Shaanxi University of Technology, \\ Hanzhong 723001, P.R. China. \\ 2. Centre of Molecular and Environmental Biology University of Minho, Department of Biology, Campus de Gualtar, Braga, 4710-057, Portugal.
}

$\triangle$ Corresponding author: Dr. Xiaoying Zhang. Chinese-German Joint Laboratory for Natural Product Research, College of Biological Science and Engineering, Shaanxi University of Technology, Hanzhong 723001, China. Tel: +86 916264 1011; E-mail: zhxying@snut.edu.cn; zhang@bio.uminho.pt

(c) The author(s). This is an open access article distributed under the terms of the Creative Commons Attribution License (https://creativecommons.org/licenses/by/4.0/). See http:/ /ivyspring.com/terms for full terms and conditions.

Received: 2019.07.18; Accepted: 2019.12.08; Published: 2020.02.04

\begin{abstract}
Acetaminophen (APAP) and roxithromycin (ROX) are often used in combination in clinical practice. To evaluate their drug-drug interactions (DDIs) and the hepatotoxicity of co-administration, rats were randomly separated into four groups: Control, APAP (50 mg/kg), ROX (5.5 mg/kg) and APAP-ROX (50 $\mathrm{mg} / \mathrm{kg}$ and $5.5 \mathrm{mg} / \mathrm{kg}$, respectively). The pharmacokinetic parameters between APAP and ROX were assayed by HPLC, and a cocktail method was used to evaluate the activities of cytochrome (CYP) 450. The liver microsome CYP2El protein was detected using Western blot. The levels of plasma parameters, mRNA levels of inflammatory factors (TNF- $\alpha$, INF- $y$, VCAM-1, CXCL-1 and STAT-3) and antioxidant factors (Nrf-2, GSTA, GCLC-1, HO-1 and NQO1) were determined using real-time PCR, along with the observation on histopathological changes in the liver tissue. APAP and ROX co-treatment significantly increased CYP2E1 activity, decreased CYP2D6 activity and prolonged APAP and ROX clearance. Co-treatment increased mRNA expressions of TNF- $\alpha, N Q O 1$ and MDA while decreasing GPX and SOD levels. Histopathological evidence showed the changes of liver tissues in terms of structure, size and tight arrangement. This study confirmed that a combination of APAP and ROX inhibited APAP metabolism and that the peak concentration of ROX was delayed. The resulting high level of CYP2E1 may induce oxidative stress and cause liver damage.
\end{abstract}

Key words: acetaminophen (APAP), roxithromycin (ROX), cytochrome P450 (CYP450), drug-drug interactions (DDIs), hepatotoxicity

\section{Introduction}

Drug combinations may enhance the therapeutic effect of individual drugs, while drug-drug interactions (DDIs) are generally unfavourable. Co-administration may alter the drug-handling capacity of each drug, leading to modified clearance, enhanced side effects and failure of drug therapy [1]. The primary focus in pharmacokinetic DDIs is the cytochrome P450 (CYP450) enzyme family. This is because of their promiscuity and prevalence in the metabolism of many drugs and xenobiotics, as many chemicals have been identified as inhibitors or inducers of CYP450, majorly in the liver, including CYP2E1, CYP1A2, CYP2D6 and CYP3A4 [2].
Inhibition or induction of CYP450 enzymes has been recognised as the determinant of metabolic drug interactions and as significantly increasing the risk of serious adverse reactions or curative failure [3].

Drug metabolites may be an important factor in hepatotoxicity. Recent studies have shown that the incidence of drug-induced liver injury (DILI) in China is at least $23.80 / 100,000$ [4], which is particularly striking when compared to the incident rate of $2.7 / 100,000$ in the US and the rate ranging from $1 / 100,000$ to $20 / 100,000$ in Europe $[5,6]$. The most important drugs causing liver injury in China are various health products and traditional Chinese 
medicine $(26.81 \%)$, anti-TB drugs (21.99\%), anticancer drugs or immune adjustment agents (8.34\%) [4].

Acetaminophen (APAP) is a widely used analgesic and antipyretic drug [7]. Roxithromycin (ROX) is used for the treatment of upper respiratory tract infections, as well as skin and soft tissue infections caused by bacteria [8]. This study aimed to investigate the drug-drug interactions of a mixture of APAP and ROX on hepatotoxicity by evaluating CYP450.

\section{Materials and Methods}

\section{Animals and treatments}

A total of 40 eight-week-old inbred SpragueDawley (SD) female rats $(260 \pm 20 \mathrm{~g})$ were purchased from College of Medicine, Xi'an Jiaotong University (Xi'an, China). All rats were maintained in a conventional sanitary facility, with the required consistent temperature and relative humidity. All experimental animal protocols were reviewed and approved by the institutional Ethics Committee for the use of laboratory animals.

\section{APAP and ROX on CYP450 activities}

Rats were randomly divided into four groups of five $(n=5)$ : normal control $(\mathrm{NC}, 0.9 \%$ physiological saline); APAP (50 mg/ $\mathrm{kg})$, ROX (5.5 mg/ $\mathrm{kg}$ ) and APAP-ROX (50 mg/ kg APAP and $5.5 \mathrm{mg} / \mathrm{kg}$ ROX). The drugs were intragastrically administrated twice per day to each group for three days. After consecutive oral administration for three days, a cocktail solution at a dose of $5 \mathrm{~mL} / \mathrm{kg}$, which contained chlorzoxazone $(20 \mathrm{mg} / \mathrm{kg})$ in a CMC-Na solution, was administered orally to each group. Pre-dose was started at $0 \mathrm{~h}$, followed $0.167,0.25,0.5,1$, 2, 4, 6, 12, 24 and $36 \mathrm{~h}$. The blood samples were collected and centrifuged at $5939 \mathrm{~g}$ for $10 \mathrm{~min}$. Plasma samples were collected and stored at $-80^{\circ} \mathrm{C}$ until use.

\section{Liver injury investigation on rats}

As in the CYP450 effects procedure, the rats were randomly divided into four groups of five $(n=5)$ : normal control (NC, $0.9 \%$ physiological saline), APAP $(50 \mathrm{mg} / \mathrm{kg})$, ROX $(5.5 \mathrm{mg} / \mathrm{kg})$ and APAP-ROX (50 $\mathrm{mg} / \mathrm{kg}$ APAP and $5.5 \mathrm{mg} / \mathrm{kg}$ ROX). The drugs were intragastrically administrated twice per day to each group for three days. The animals were weighed and sacrificed to collect blood and liver tissue samples. The serum was separated from the blood samples by centrifugation at $900 \mathrm{~g}$ and $4^{\circ} \mathrm{C}$ for $10 \mathrm{~min}$ and stored at $-20^{\circ} \mathrm{C}$ until further analysis.

\section{Chromatographic conditions}

Analyses were performed with a 1260 series liquid chromatographer (Agilent, Santa Clara, USA) equipped with a quaternary pump, an autosampler and a thermostatted column compartment (Phenomenex, Torrance, USA). Chromatographic separation was achieved on a $100 \mathrm{~mm} \times 4.6 \mathrm{~mm}, 3.5 \mu \mathrm{m}$ particle Agilent Zorbax Eclipse Plus-C18 columns at $25^{\circ} \mathrm{C}$. The mobile phase consisted of $0.1 \%$ formic acid in water (A) and acetonitrile (B) in elution as follows: A:B (85:15). The detection wavelength was $249 \mathrm{~nm}$ for APAP and $0.1 \mathrm{~mol} / \mathrm{L}$ ammonium dihydrogen phosphate $(\mathrm{pH} 6.5 \mathrm{~A})$ and acetonitrile $(\mathrm{B})$ in elution as follows: A:B (65:35). The detection wavelength was $230 \mathrm{~nm}$ for ROX, the flow rate was $0.8 \mathrm{~mL} / \mathrm{min}$, the column temperature was $25^{\circ} \mathrm{C}$, and the typical injection volume was $20 \mu \mathrm{L}$.

\section{HPLC sample preparation}

All plasma samples were homogenised with acetonitrile. After centrifugation at $5939 \mathrm{~g}$ and $4^{\circ} \mathrm{C}$ for $10 \mathrm{~min}$, the supernatant was stored at $4^{\circ} \mathrm{C}$ for $24 \mathrm{~h}$, and centrifuged again under the same conditions. The supernatant was filtered through a $0.22 \mu \mathrm{m}$ filter, and $20 \mu \mathrm{L}$ of the sample filter liquid was injected into the HPLC system for analysis. The standard curve consisted of samples containing 3.125, 6.25, 12.5, 25 and $50 \mu \mathrm{g} / \mathrm{mL}$ of APAP and 12.5, 25, 50, 100, 125 and $250 \mu \mathrm{g} / \mathrm{mL}$ of ROX.

\section{Pharmacokinetic analysis}

Model fitting and evaluation of the pharmacokinetic parameters were carried out using Drug and Statistics 2.0 (DAS); pharmacokinetic parameters were determined using the non-compartmental method based on the statistical moment theory.

\section{Serum ALT and AST assays}

Enzymatic activities of serum alanine aminotransferase (ALT) and aspartate aminotransferase (AST) were estimated spectrophotometrically using commercial diagnostic kits (Jiancheng Institute of Biotechnology, Nanjing, China).

\section{Hepatic MDA, SOD and GPX determinations}

Frozen liver tissues were thawed and homogenised in ice-cold PBS. The homogenate was centrifuged at $900 \mathrm{~g}$ for $10 \mathrm{~min}$ at $4^{\circ} \mathrm{C}$, and the supernatants were assayed for malondialdehyde (MDA), superoxide dismutase (SOD) and glutathione peroxidase (GPX) levels using the commercial assay kits, as per manufacturer's instructions (Jiancheng Institute of Biotechnology, Nanjing, China). The protein concentrations in tissue homogenates were measured by Bradford protein assay using bovine serum albumin (BSA) as the standard (Tiangen Biotech, Beijing, China). 
Table 1. Primers used for real-time quantitative PCR

\begin{tabular}{|c|c|c|}
\hline Target gene & Forward primer $\left(5^{\prime}-3^{\prime}\right)$ & Reverse primer $\left(5^{\prime}-3^{\prime}\right)$ \\
\hline TNF- $\alpha$ & TATGGCTCAGGGTCCAACTC & GCTCCAGTGAATTCGGAAAG \\
\hline IFN- $\gamma$ & $\begin{array}{l}\text { TCAAGTGGCATAGATGTGGA } \\
\text { AGAA }\end{array}$ & TGGCTCTGCAGGATTTTCATG \\
\hline VCAM-1 & AGCCTCAACGGTACTTTGGA & GCGTTTAGTGGGCTGTCTAT \\
\hline CXCL-1 & $\begin{array}{l}\text { GATTCACCTCAAGAACATCC } \\
\text { AGAG }\end{array}$ & GAAGCCAGCGTTCACCAGAC \\
\hline STAT-3 & TGCAGAGCAGGTATCTTGAG & TGCTGCTTCTCTGTCACTAC \\
\hline Nrf-2 & $\begin{array}{l}\text { GCTGATGGAGTACCCTGAGG } \\
\text { CTAT }\end{array}$ & $\begin{array}{l}\text { ATGTCCGCAATGGAGGAGA } \\
\text { AGTCT }\end{array}$ \\
\hline GSTA-2 & $\begin{array}{l}\text { TCAGTAACCTGCCCACAGTG } \\
\text { AAGA }\end{array}$ & $\begin{array}{l}\text { GCATGTTCTTGACCTCTATGG } \\
\text { CTGG }\end{array}$ \\
\hline GCLC-1 & TGAGATTTAAGCCСССТCCT & TTGGGATCAGTCCAGGAAAC \\
\hline $\mathrm{HO}-1$ & $\begin{array}{l}\text { TGCCAGTGCCACCAAGTTCA } \\
\text { AG }\end{array}$ & $\begin{array}{l}\text { TGTTGAGCAGGAACGCAGTC } \\
\text { TTG }\end{array}$ \\
\hline NQO1 & $\begin{array}{l}\text { GGAGACAGCCTCTTACTTGC } \\
\text { CAAG }\end{array}$ & $\begin{array}{l}\text { CCAGCCGTCAGCTATTGTGG } \\
\text { ATAC }\end{array}$ \\
\hline$\beta$-actin & $\begin{array}{l}\text { CGTTGACATCCGTAAAGACC } \\
\text { TC }\end{array}$ & $\begin{array}{l}\text { TAGGAGCCAGGGCAGTAATC } \\
\mathrm{T}\end{array}$ \\
\hline
\end{tabular}

Table 2. The pharmacokinetic parameters of APAP and ROX

\begin{tabular}{lllll}
\hline Parameter & APAP & Co-treatment & ROX & Co-treatment \\
\hline $\mathrm{T}_{1 / 2 \mathrm{z}}(\mathrm{h})$ & $4.74 \pm 1.00$ & $19.10 \pm 0.63^{*}$ & $4.07 \pm 3.20$ & $3.05 \pm 2.56$ \\
$\mathrm{~T}_{\max }(\mathrm{h})$ & $0.56 \pm 0.41$ & $1.00 \pm 0.26$ & $3.00 \pm 0.88$ & $1.00 \pm 0.37 \#$ \\
$\mathrm{C}_{\max }(\mathrm{mg} / \mathrm{L})$ & $3.91 \pm 0.99$ & $3.44 \pm 0.68$ & $93.64 \pm 52.56$ & $64.12 \pm 42.75^{\#}$ \\
$\mathrm{MRT}(0-\infty)(\mathrm{h})$ & $5.97 \pm 1.10$ & $20.64 \pm 1.21$ & $5.80 \pm 4.78$ & $4.99 \pm 3.21$ \\
$\mathrm{AUC}(0-\infty)$ & $27.11 \pm 4.00$ & $15.45 \pm 2.63^{*}$ & $796.37 \pm 452.68$ & $391.64 \pm 83.41^{\#}$ \\
$(\mathrm{mg} / \mathrm{h} / \mathrm{L})$ & & & & $0.051 \pm 0.061$ \\
$\mathrm{CLz}(\mathrm{L} / \mathrm{h} / \mathrm{kg})$ & $1.05 \pm 0.43$ & $1.294 \pm 0.32$ & $0.025 \pm 0.040$ & $0.05) ; \#$ \\
\multicolumn{5}{l}{$\begin{array}{l}\text { Note: }{ }^{*} \text { refers to the significant difference of APAP group to control }(p<0.05) \\
\text { refers to the significant difference of ROX group to control }(p<0.05) .\end{array}$}
\end{tabular}

\section{Histological analysis}

Liver tissues were fixed in $10 \%$ formalin and embedded in paraffin for histological assessment. Samples were subsequently sectioned $(5 \mathrm{~mm})$, stained with haematoxylin and eosin (H\&E) and examined using bright field light microscopy (Olympus, Tokyo, Japan).

\section{Western blot analysis}

The protein concentration was determined using the Bradford protein assay (Tiangen Biotech, Beijing, China). Proteins were resolved by SDS-PAGE and transferred to PVDF membranes (Millipore Corporation, Boston, ME, USA). After blocking in TBST containing 5\% skimmed milk powder, the membranes were incubated overnight at $4^{\circ} \mathrm{C}$ with primary antibodies against cleaved CYP2E1 (1:500; Boster, Wuhan, China) and glyceraldehyde phosphate ehydrogenase (B-actin, 1:1000; Tianjin Sungene Biotech, Tianjin, China). Blots were then incubated with a 1:2000 dilution of horseradish peroxide conjugated secondary antibodies (Sungene Biotech, Tianjin, China) for $2 \mathrm{~h}$ at room temperature. Protein bands were visualised by ECL reaction (Genshare Biological, Xi'an, China). The protein levels were quantified using Gel-Pro Analyzer software (Media Cybernetics, Washington, MD, USA).

\section{Determination of the inflammation-related gene and antioxidant factor expressions by real-time quantitative PCR}

The mRNA was isolated from the frozen liver tissues using a Total RNA isolation kit (Tiangen Biotech, Beijing, China), according to the manufacturer's instructions. The mRNA samples were then reverse-transcribed into cDNA using HiScript $^{\mathrm{TM}}$ RT SuperMix for Real-Time Polymerase Chain Reaction (qPCR; Vazyme Biotech, Nanjing, China). The aliquots of cDNAs were amplified using specific primers (Table 1). The real-time PCR was performed on a QuantStudio ${ }^{\mathrm{TM}} 6$ Flex Real-Time PCR System (Life Technologies, MD, USA) with SYBR ${ }^{\circledR}$ Green Master Mix (Vazyme Biotech, Nanjing, China). The relative expression of mRNA was expressed by the $2-(\Delta \Delta \mathrm{Ct})$ formula and normalised to that of $\beta$-actin, an internal control gene.

\section{Statistical analysis}

All the experimental data were expressed as mean \pm SD. The significant difference from the respective control in all experiments was assessed by one-way analysis of variance (ANOVA) using SPSS (IBM Corporation, Chicago, USA). Values of $p<0.05$ were considered statistically significant.

\section{Results}

\section{Pharmacokinetic analysis of APAP and ROX}

The concentration of the APAP and ROX standard solution $(\mu \mathrm{g} / \mathrm{mL})$ was plotted on the abscissa. The standard working curve of APAP as $\mathrm{Y}=135.53 \mathrm{x}+$ $6.5144\left(\mathrm{R}^{2}=0.999\right)$ and of $\mathrm{ROX}$ as $\mathrm{Y}=3.9328 \mathrm{x}+21.97$ $\left(\mathrm{R}^{2}=0.992\right)$ provided peak areas of each component to be plotted on the ordinate. The pharmacokinetic parameters of APAP and ROX were determined by HPLC, and the $C_{\max }$ of the APAP and ROX co-treatment was significantly increased compared to the separated APAP or ROX treatment (Table 2). The AUC of APAP and ROX co-treatment was significantly reduced compared to either APAP or ROX alone (Figure 1).

\section{Co-treatment effects of APAP and ROX on CYP2D6 and CYP2E 1}

The standard solution concentration $(\mu \mathrm{g} / \mathrm{mL})$ of the cocktail substrate (chlorzoxazone and dextromethorphan) was taken as the abscissa. The standard working curves of chlorzoxazone as $Y=19.328 x+18.6$ $\left(R^{2}=0.999\right)$ and dextromethorphan as $Y=25.927 x-$ $9.1585\left(R^{2}=0.99\right)$ were both plotted on the ordinate.

The pharmacokinetic effect of CYP450 was compared with the cocktail probe drug method in the APAP and ROX co-treatment group. The activity of 
CYP2E1 and CYP2D6 accelerated the metabolism of chlorzoxazone and decelerated that of dextromethorphan, respectively. The co-treatment of APAP and ROX had different effects on the CYP450 subtypes, namely inducing CYP2E1 and inhibiting CYP2D6 (Figure 2).

\section{Co-treatment of APAP and ROX on hepatic dysfunction}

Microscopic analysis of tissue sections of rats from the APAP, ROX and APAP-ROX co-treatment groups revealed considerable changes from co-treatment (Figure 3). The liver cells showed pathological changes in loose arrangement, extravasated blood and vascular degeneration (Figure 3D); further, serum ALT and AST activities were significantly increased in the co-treatment group compared with the NC group (Figure 4).

\section{Co-treatment of APAP and ROX on liver oxidative stress}

The co-administration of APAP-ROX resulted in significantly increased levels of MDA and depleted GPX and SOD activity levels. Simultaneously, the chemokine mRNA levels were analysed by Q-RTPCR. The levels of Nrf-2, GSTA, GCLC-1, HO-1 and NQO1 had no significant differences in the APAP group and

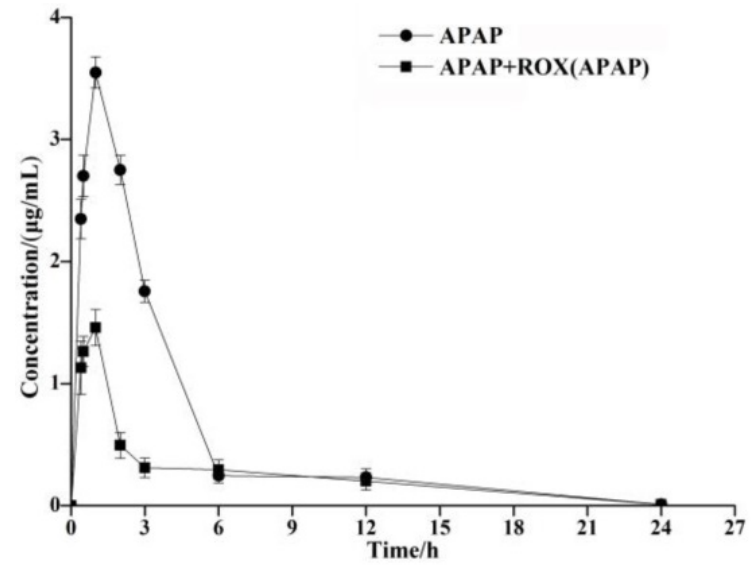

ROX group compared with the NC group ( $p>0.05$; Figure 6), suggesting that a single administration of APAP and ROX did not induce inflammation. In contrast, in the co-treatment group, the expression of those chemokine mRNA markedly increased compared to the NC group ( $p<0.05)$, indicating that co-treatment of APAP and ROX induced liver injury and increased the activities of Nrf-2, GSTA-2, GCLC-1, HO-1 and NQO1 (Figure 6).

\section{Determination of CYP2E1 expressions on APAP and ROX}

The expressions of the CYP2E1 protein were markedly increased in the APAP and ROX groups $(p<$ $0.05)$, particularly in the co-treatment group, when compared to the NC group ( $p<0.01$; Figure 7).

\section{The effect of APAP and ROX on liver inflammation}

The levels of TNF- $\alpha$, INF- $\gamma$, VCAM-1, CXCL-1 and STAT-3 were not significantly different in the APAP group and ROX group expression levels compared with the NC group. The levels of hepatic TNF- $\alpha$, INF- $\gamma$, VCAM-1, CXCL-1 and STAT-3 significantly increased co-treatment of the APAP and ROX groups compared with the NC group $(p<0.05$; Figure 8).

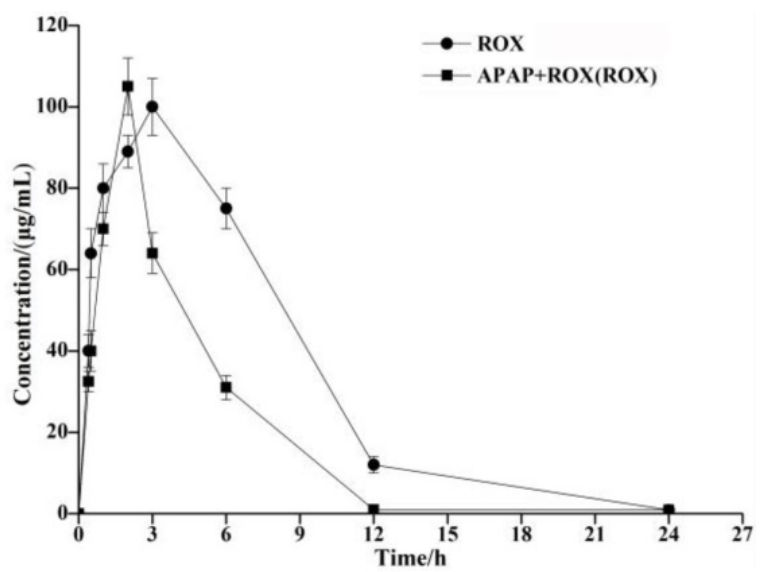

Figure 1. Time-concentration curves of APAP and ROX in rats. The AUC of APAP and ROX co-treatments was significantly reduced compared to either APAP or ROX alone.
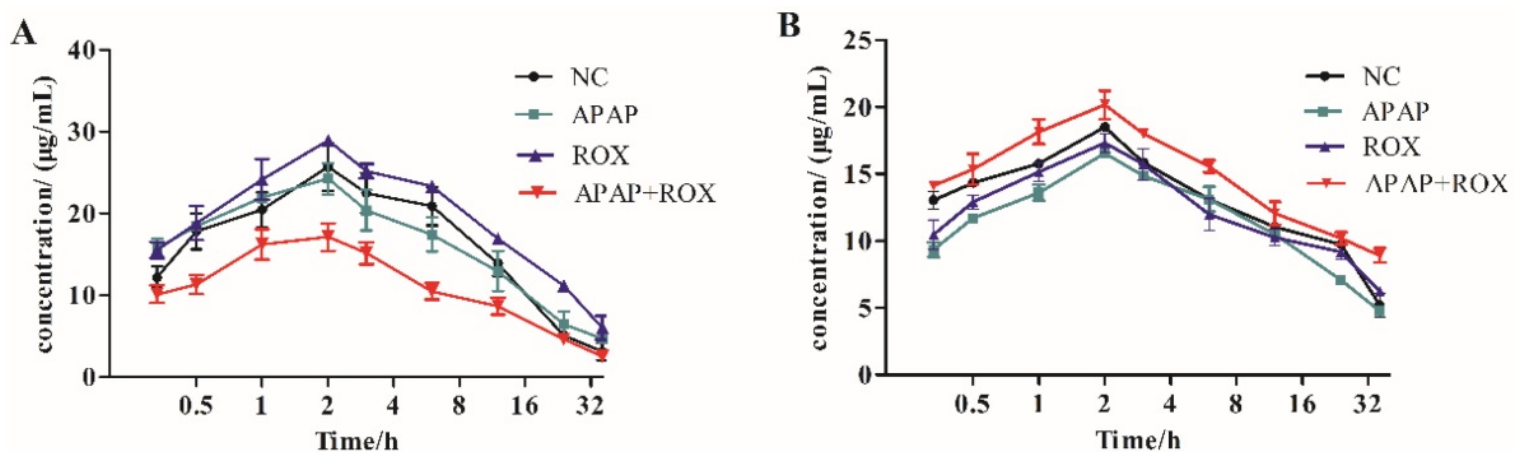

Figure 2. Time-concentration curves. Substrate inductions: (A) chlorzoxazone; (B) dextromethorphan; mean + SD ( $n=4)$. The co-treatment of APAP and ROX had different effects on the CYP450 subtypes, which induced CYP2E1 and inhibited CYP2D6. 


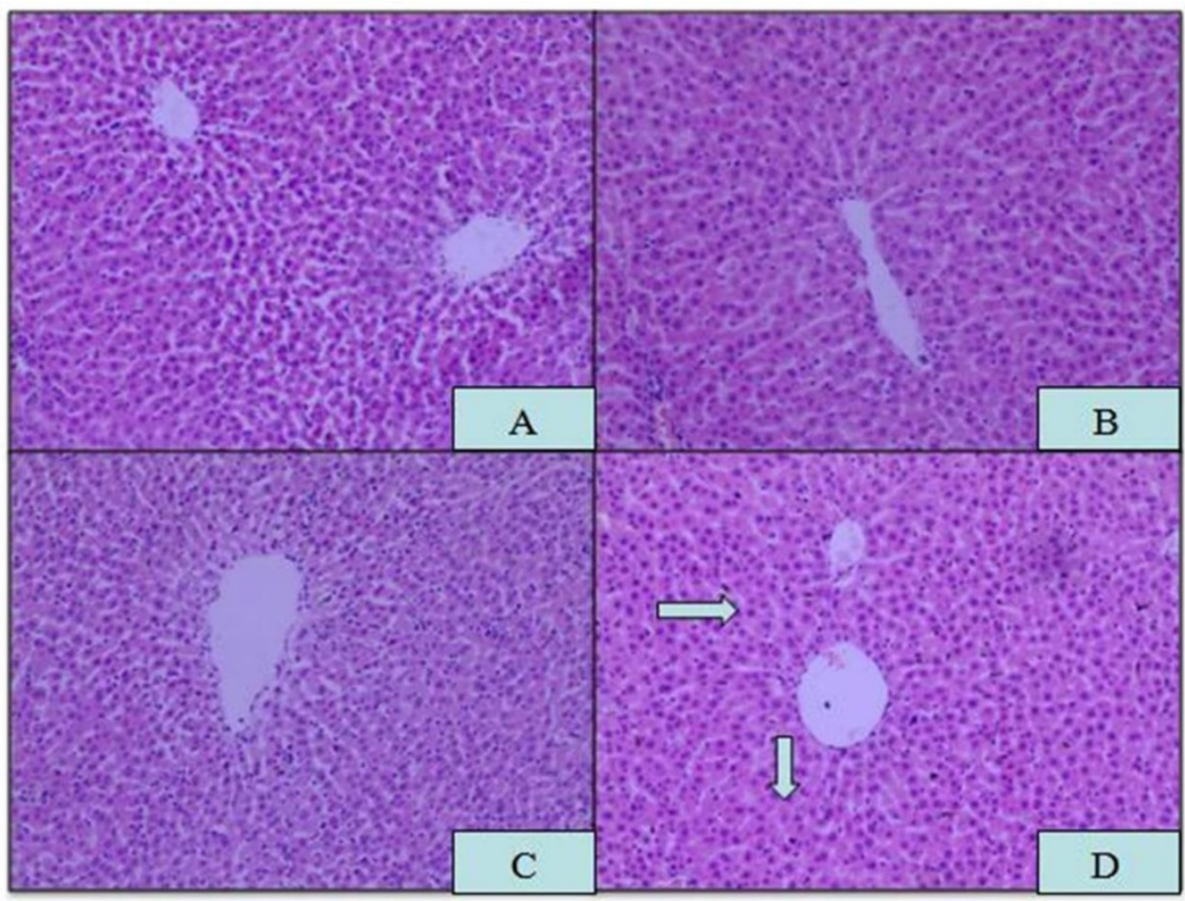

Figure 3. Histopathological changes in liver tissue. Magnification: 10×10; (A) NC; (B) APAP; (C) ROX; (D) APAP-ROX. $\rightarrow$ Dissolution of the nucleus. The liver cells showed pathological changes in loose arrangement, extravasated blood and vascular degeneration (D).
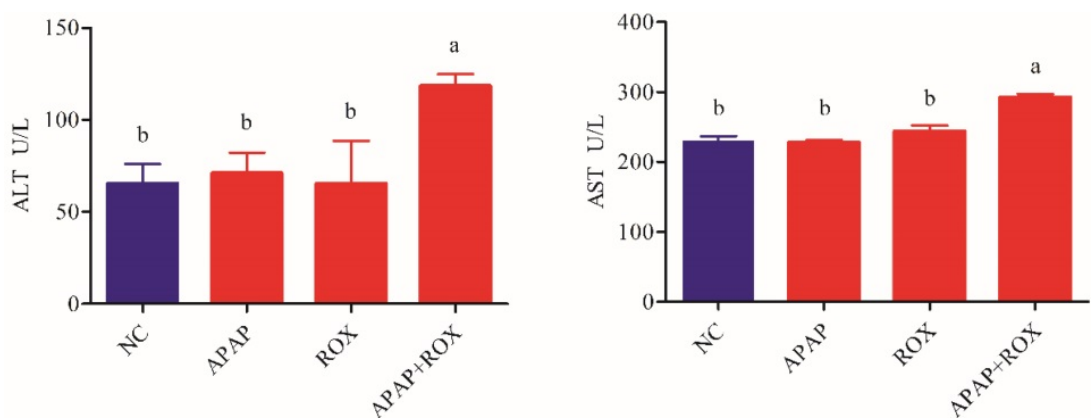

Figure 4. Increased ALT and AST in APAP-ROX co-treatment. Each value represents the mean \pm SD of three independent experiments; labels a-d indicate statistically different groups $(p<0.05)$. The serum ALT and AST activities of APAP-ROX co-treatment groups were significantly increased in the co-treatment group compared with the NC group.
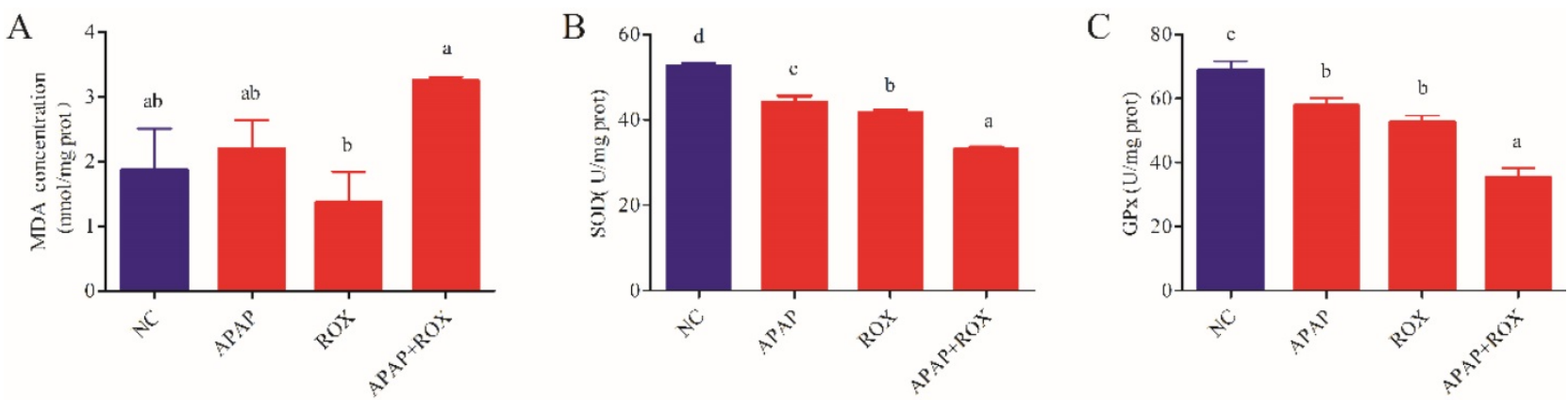

Figure 5. The oxidative changes in liver tissue. Notes: Each value represents the mean \pm SD of three independent experiments; labels a- $d$ indicate statistically different groups $(p<0.05)$. The APAP and ROX co-treatment increased the MDA level and depressed GPX and SOD activities.

\section{Discussion}

Drug-drug interactions are usually unfavourable. DDIs affect the metabolism of each drug in the body, which may change CYP450 enzyme activity. In normal conditions, APAP is predominantly metabolised in the liver by conjugating with glucuronic acid and sulphate [9]. Overdosed APAP is metabolised by CYP2E1, which catalyses two-electron oxidation to reactive and toxic $\mathrm{N}$-acetyl-p-benzoquinone imine (NAPQI) and induces oxidative stress, mitochondrial dysfunction, inflammation and DNA damage [10]. The hepatotoxicity induced by APAP exhibited a 
circadian rhythm with the peak liver toxicity when administrated (injection) at evening (20:00), while with markedly decreased liver damage when the administration is conducted at morning (08:00). Furthermore, the circadian rhythms may be associated with the expression of hepatic glutathione (GSH) [11] under the mediation of clock gene such as mPer2 [12]. In the current study, the APAP and ROX combination resulted in prolonged clearance of APAP (Table 2) and enhanced APAP toxicity.

Cellular CYP2E1 is known to mediate prolonged alcohol and APAP induced toxicity in hepatic and extra-hepatic cells. Chronic ethanol intake may enhance APAP toxicity by producing a persistent up-regulation of CYP2E1, as well as depleting GSH stores $[13,14]$. In the current study, APAP and ROX were administrated to rats, with the dose calculated to be standard through conversion of human body surface area to rat body surface area. The APAP-ROX co-treatment increased CYP2E1 activity and decreased CYP2D6 activity in rat hepatocytes (Figure 2 ). Consequently, the combined management of APAP-ROX resulted in the long-term clearance of APAP and ROX (Table 2).
A

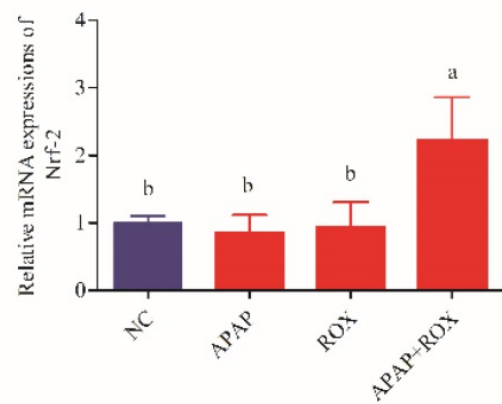

D

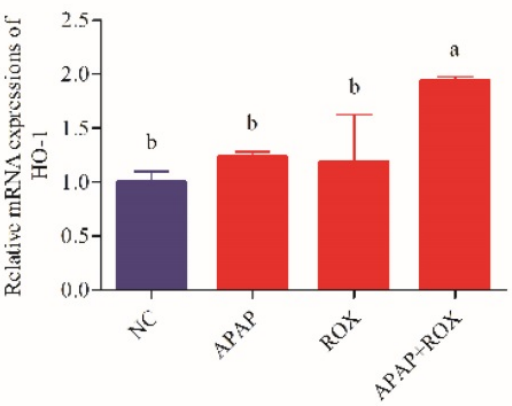

B

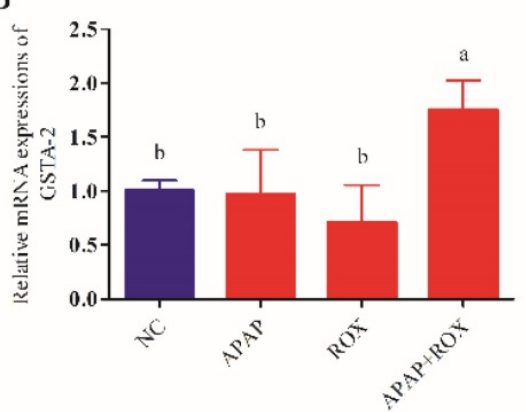

E

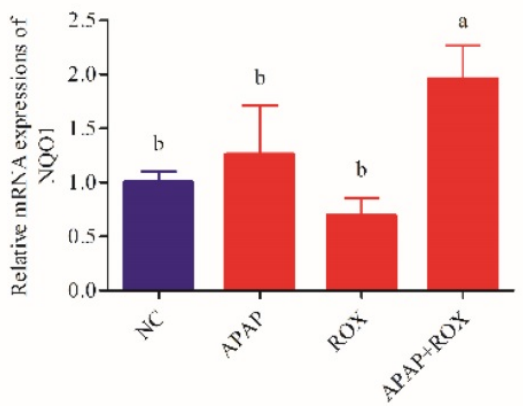

$\mathrm{C}$

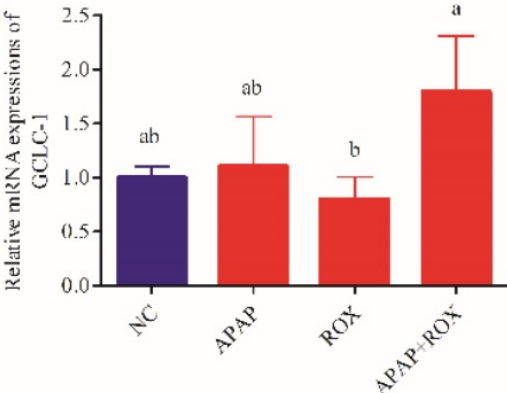

Figure 6. Effect of APAP-ROX on the chemokine mRNA expression levels. Each value represents the mean \pm SD of three independent experiments; labels a-d indicate statistically different groups ( $p<0.05)$. The levels of Nrf-2, GSTA, GCLC-1, HO-1 and NQO1 had no significant differences in the APAP group and ROX group compared with the NC group. In the co-treatment group, the expression of those chemokine mRNA markedly increased compared to the NC group.

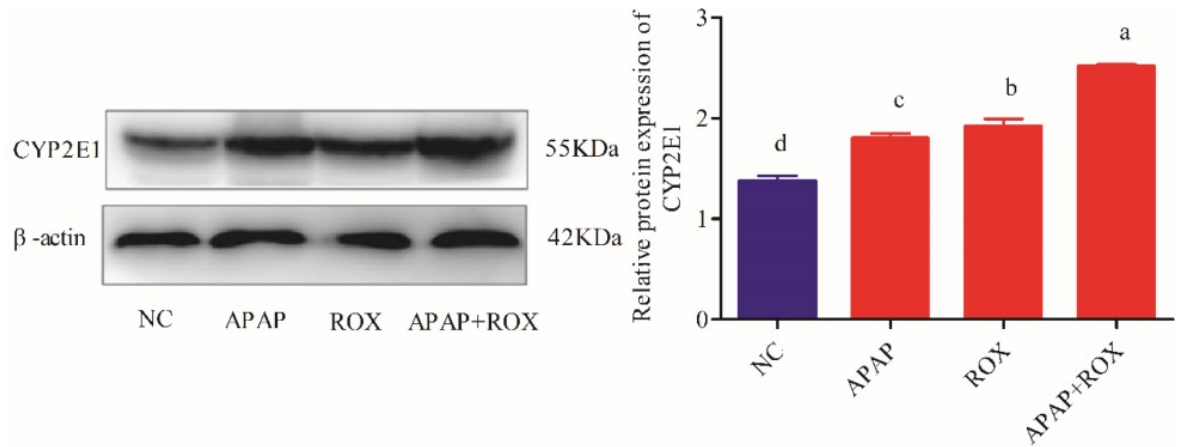

Figure 7. Liver CYP2E1 expression. Each value represents the mean \pm SD of three independent experiments; labels a-d indicate statistically different groups ( $p<0.05$ ). The expressions of the CYP2E1 protein were markedly increased in the APAP and ROX groups $(p<0.05)$, particularly in the co-treatment group, when compared to the NC group. 

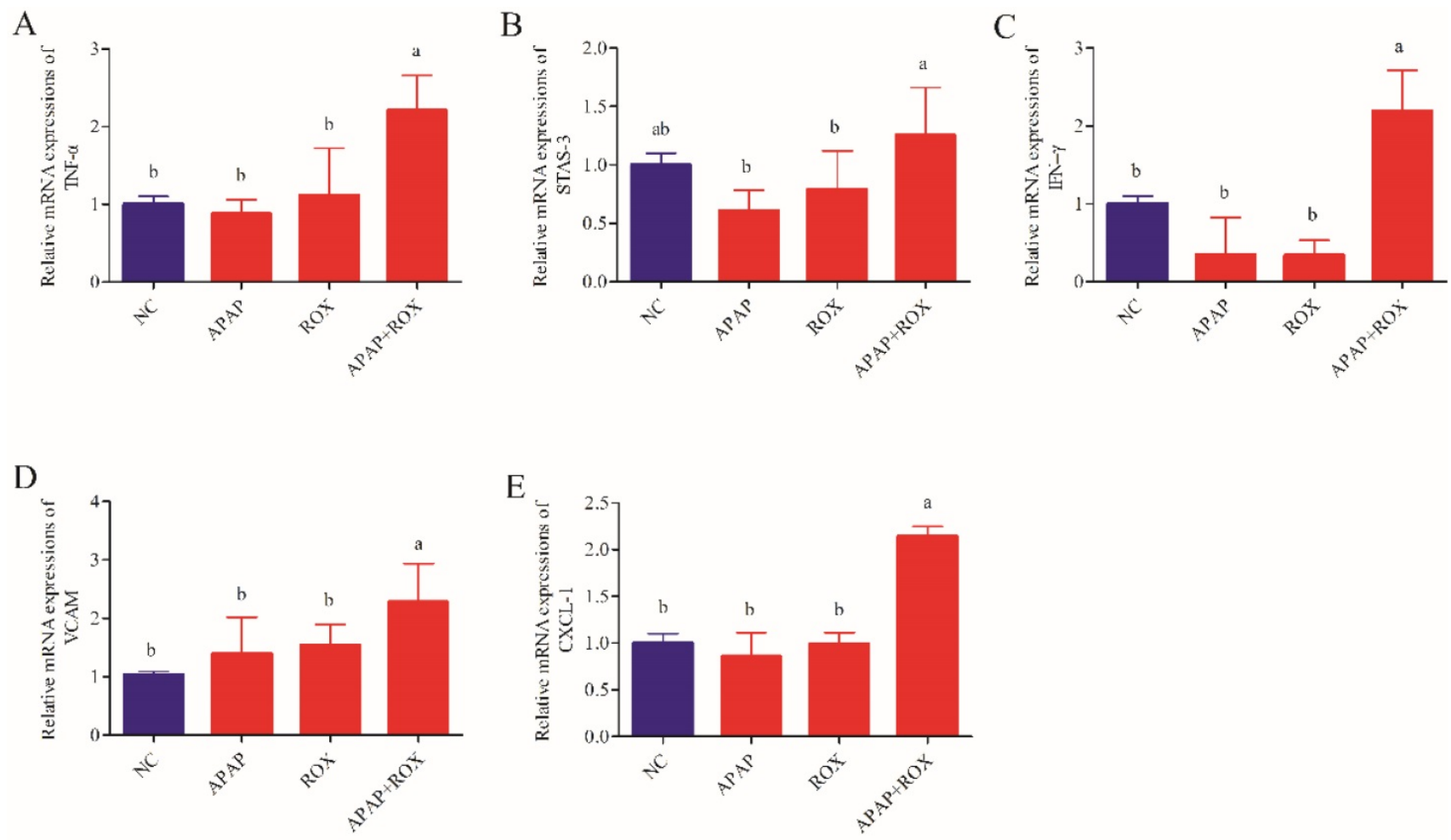

Figure 8. The expression level of inflammation factors mRNA. Each value represents the mean \pm SD of three independent experiments; labels a- $d$ indicate statistically different groups $(p<0.05)$. The levels of hepatic TNF- $\alpha$, INF-y, VCAM-1, CXCL-1 and STAT-3 significantly increased co-treatment of the APAP and ROX groups compared with the NC group.

Clinically, the activities of AST and ALT are regarded as sensitive indicators of hepatotoxicity [15]. The hepatoxicity was observed in the rat after APAP and ROX co-treatment, as characterised by higher levels of serum ALT, AST and hepatic histopathological lesions.

Healthy human cells have an effective anti-oxidative and anti-inflammatory defence system, in which SOD and GPX are the main enzymes [16]. When drug intake is excessive, the body's scavenging capacity changes, and oxidative damage occurs in the liver [17]. The current study confirmed that an APAP and ROX co-treatment increased the MDA level and depressed GPX and SOD activities (Figure 5), suggesting the impairment of hepatic redox homeostasis, accumulation of ROS and formation of lipid peroxidation. Co-treatment significantly increased the mRNA expression of Nrf2 (Figure 6), which is significant because the activated Nrf2 is a transcription factor that modulates endogenous antioxidant enzymes [18, 19]. APAP and ROX stimulate Nrf2 activation, which binds to the antioxidant response element and further activates the transcription of gene-encoding for antioxidants and detoxification, including haem oxygenase-1 (HO-1), NADPH, quinone oxidoreductase-1 (NQO-1) and glutathione-synthesising enzymes glutamatecysteine ligase catalytic subunit (GCLC). The results suggest that increased mRNA expression of Nrf2 after co-treatment can lead to transcriptional activation of antioxidant enzymes (HO-1, SOD and CAT; Figure 6).

Drug candidates that cause intrinsic liver injury are usually weeded out in preclinical testing, the intrinsic reaction of APAP are predictable, dosedependent, and usually occur in overdose settings or with pre-existing hepatic impairment. Idiosyncratic drug-induced liver injury could be widely existed among pharmaceutical products, but with limited confirmed information. It is interesting and necessary to further investigate whether ROX may lead to an idiosyncratic reaction, and whether it is dosage, patient or environmental risk related [20, 21], especially in the content of drug-drug interactions. ROS-mediated inflammation plays a vital role in the pathogenesis of APAP [22]. During the inflammation caused by APAP and ROX overdose, the cytokines were up-regulated and accumulated in the liver. Among these, TNF- $\alpha$ and Nrf-2 have been recently implicated as critical mediators of APAP-induced hepatotoxicity $[23,24]$. Our study demonstrated that APAP and ROX co-treatment significantly increased the expression levels of TNF- $a$, INF- $\gamma$, VCAM-1, CXCL-1, STAT-3, Nrf-2, GSTA, GCLC-1, HO-1 and NQO1, suggesting that APAP and ROX co-treatment can induce liver inflammation.

In summary, the combination of APAP and ROX caused metabolic changes in each drug and led to a certain degree of liver damage, as co-treatment 
inhibited the activities of CYP2D6 and increased the expressions and activities of CYP2E1, which led to slower APAP eliminations and higher drug plasma levels, thereby inducing oxidative stress and hepatotoxicity.

\section{Acknowledgements}

This study was supported by the National Key Research and Development Program of China (2017Y FD0501405); Incubation Project on State Key Laboratory of Biological Resources and Ecological Environment of Qinba Areas (SLGPT2019KF04-04); Research Project of Shaanxi Provincial Education Department (17JK0137); Innovation Capability Support Program of Shaanxi (2019XY-04), China and UID/BIA/04050/2013 (POCI-01-0145-FEDER-007569) funded by national funds through the FCT and the ERDF through the COMPETE2020, Portugal. We are grateful to Ms. Ruimin Cao for her technical work.

\section{Competing Interests}

The authors have declared that no competing interest exists.

\section{References}

1. Michael G, Berg AH, Solano JJ, Steven S. Inhibition of CYP2E1 with propylene glycol does not protect against hepatocellular injury in human acetaminophen daily-dosing model. J Clin Pharmacol. 2018; 59:131-8.

2. Polasek TM, Lin FPY, Miners JO, Doogue MP. Perpetrators of pharmacokinetic drug-drug interactions arising from altered cytochrome P450 activity: a criteria-based assessment. Brit J Clin Pharmac. 2011; 71: 727-36.

3. Brown CM, Reisfeld B, Mayeno AN. Cytochromes P450: a structure-based summary of biotransformations using representative substrates. Drug Metab Rev 2008; 40: 92-100.

4. Cheng Y, Liang X, Feng L, Liu D, Qin M, Liu S, et al. Effects of phillyrin and forsythoside $\mathrm{A}$ on rat cytochrome $\mathrm{P} 450$ activities in vivo and in vitro. Xenobiotica. 2017; 47: 297-303.

5. Shen TL, Shang J, Xie O, Li J, Yan M, Xu J, et al. Incidence and Etiology of Drug-Induced Liver Injury in Mainland China. Gastroenterology. 2019; 156: 2230-41.

6. Watkins PB, Seeff LB. Drug-induced liver injury: summary of a single topic clinical research conference. Hepatology. 2010; 43: 618-31.

7. Bell LN, Naga C. Epidemiology of idiosyncratic drug-induced liver injury. Semin Liver Dis. 2009; 29: 337-47.

8. Björnsson E. Review article: drug-induced liver injury in clinical practice. Aliment Pharm Therap. 2010; 32: 3-13.

9. Amy S, Rebecca C, Mandy L, Bijan E. Acetaminophen: old drug, new warnings. Clev Clin J Med. 2010; 77: 19-27.

10. Xie W, Jiang Z, Wang J, Zhang X, Melzig MF. Protective effect of hyperoside against acetaminophen (APAP) induced liver injury through enhancement of APAP clearance. Chem Biol Interact. 2016; 246: 11-9.

11. Mori K, Kumano A, Kodama T, Takiguchi S, Takano N, Kumada K, et al. Evaluation of hepatic damage by reactive metabolites--with consideration of circadian variation of murine hepatic glutathione levels. J Toxicol Sci, 2014, 39(4): 537-44.

12. Kakan $\mathrm{X}$, Chen $\mathrm{P}$, Zhang J. Clock gene mPer2 functions in diurnal variation of acetaminophen induced hepatotoxicity in mice. Exp Toxicol Pathol. 2011; 63(6): 581-5

13. Hedgpeth B, Missall R, Bambaci A, Smolen M, Yavuz S, Cottrell J, et al. A Review of Bioinformatics Tools to Understand Acetaminophen-Alcohol Interaction. Medicines (Basel). 2019; 6(3). pii: E79. doi: $10.3390 /$ medicines6030079.

14. Rahman MA, Kodidela S, Sinha N, Haque S, Shukla PK, Rao R, et al. Plasma exosomes exacerbate alcohol- and acetaminophen-induced toxicity via CYP2E1 pathway. Sci Rep. 2019; 9(1): 6571-81.

15. Jiang Z, Chen C, Wang J, Xie W, Wang M, Li X, et al. Purple potato ( Solanum tuberosum L.) anthocyanins attenuate alcohol-induced hepatic injury by enhancing antioxidant defense. J Nat Med. 2016; 70: 1-9.

16. Valko M, Leibfritz D, Moncol J, Cronin MT, Mazur M, Telser J. Free radicals and antioxidants in normal physiological functions and human disease. Int J Biochem Cell Biol. 2007; 39: 44-84
17. Singh M, Kapoor A, Bhatnagar A. Oxidative and reductive metabolism of lipid-peroxidation derived carbonyls. Chem Biol Interact. 2015; 234: 261-73.

18. Hou Y, Peng S, Li X, Yao J, Xu J, Fang J. Honokiol alleviates oxidative stress-induced neurotoxicity via activation of Nrf2. Acs Chem Neurosci. 2018; 9: 3108-16

19. Liu XF, Zhou DD, Xie T, Hao JL, Malik TH, Lu CB, et al. The Nrf2 Signaling in Retinal Ganglion Cells under Oxidative Stress in Ocular Neurodegenerative Diseases. Int J Biol Sci. 2018; 14: 1090-8.

20. Iasella CJ, Johnson HJ, Dunn MA. Adverse Drug Reactions: Type A (Intrinsic) or Type B (Idiosyncratic). Clin Liver Dis. 2017; 21(1): 73-87.

21. Roth RA, Ganey PE. Intrinsic versus idiosyncratic drug-induced hepatotoxicity--two villains or one? J Pharmacol Exp Ther. 2010; 332(3): 692-627.

22. Du K, Ramachandran A, Jaeschke H. Oxidative stress during acetaminophen hepatotoxicity: Sources, pathophysiological role and therapeutic potential. Redox Bio. 2016; 10: 148-56.

23. Barman PK, Mukherjee R, Prusty BK, Suklabaidya S, Senapati S, Ravindran B. Chitohexaose protects against acetaminophen-induced hepatotoxicity in mice. Cell Death Dis. 2016; 7: e2224.

24. Zhao H, Jiang Z, Chang X, Xue H, Yahefu W, Zhang X. 4-Hydroxyphenylacetic acid prevents acute APAP-induced liver injury by increasing phase II and antioxidant enzymes in mice. Front Pharmacol. 2018; 9: 653. 